\title{
Preliminary Results of a Randomized, Prospective and Non-Inferiority Study Comparing Standard Vs. Lacrimal Dilator Facilitated Sub-Tenon's Block
}

\author{
Oya Yalcin Cok ${ }^{1}$, Muge Coban Karatas, ${ }^{2}$ Chandra M Kumar ${ }^{3}$
}

${ }^{1}$ Dept of Anesthesiology \& '2Dept of Ophthalmology ,Baskent University,School of Medicine, Adana, Turkey, 3Khoo Teck Puat Hospital, Singapore

\section{Background and aims}

Sub-Tenon's block (STB) is increasingly used for cataract surgery to reduce risk of complications related to needle blocks.1 The technique involves making a conjunctival incision with blunt forceps and scissors followed by insertion of a blunt cannula into the sub-Tenon's space. Recently, a sub-Tenon's technique has been described in which a small aperture in conjunctiva is made with a lacrimal dilator.2 This technique accelerates block performance, minimize local trauma, improve surgical field and avoid surgical incision of conjunctiva.3

We report preliminary results of a randomized, prospective, non-inferiority study comparing standard vs. lacrimal dilator-facilitated sub-Tenon's block.

\section{Methods}

- The patients undergoing elective cataract surgery enrolled into two groups to receive sub-Tenon's block

- Group WS - standard sub-Tenon's dissected with Westcott scissors

- Group LD - entry portal obtained with a lacrimal dilator (Fig 1)

- A 19G, $2.5 \mathrm{~cm}$ long sub-Tenon's curved blunt cannula was used in both groups

- All patients received $3 \mathrm{~mL} 2 \%$ lidocaine and no sedatives

- Data collection included:

- Demography

- duration of the procedure

- intraoperative chemosis

- analgesia (Visual Analogue Scale 1-10).

- akinesia (4 primary gazes)

- the quadrant/s of subconjunctival haemorrhage on the first postoperative day

- The latter four parameters were assessed by a 5 -score scale according to quadrants of the globe

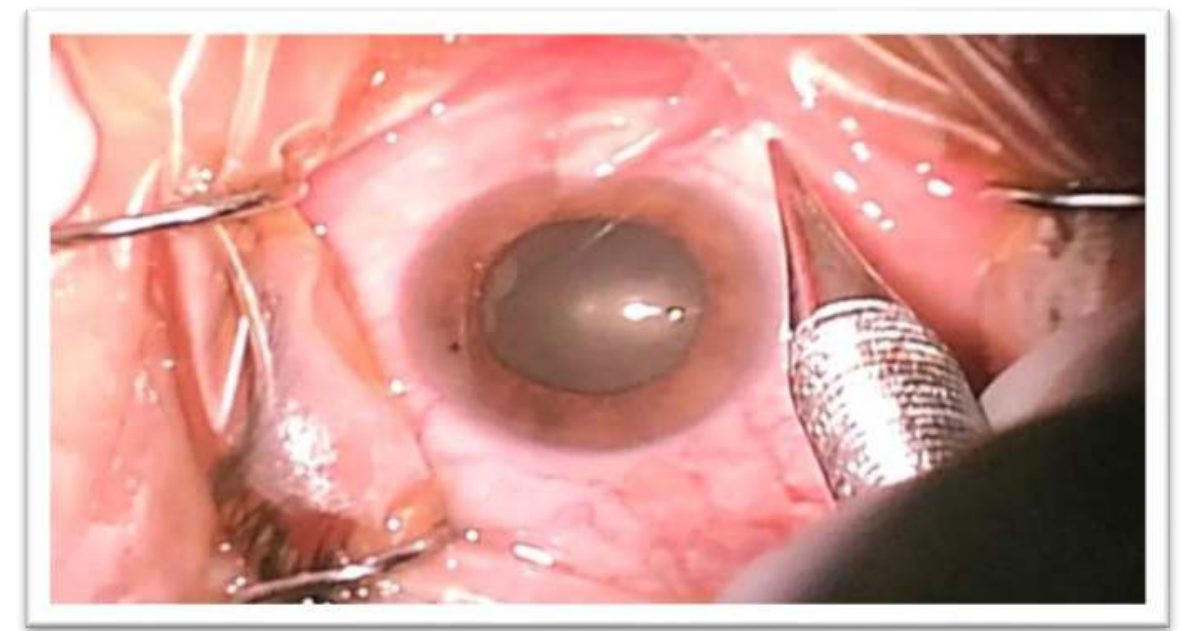

Fig1. Lacrimal dilator-facilitated entry during STB

\section{Results}

- Patients in both groups were comparable for demographic data, procedure duration, analgesia and akinesia( $p>0.05)$.

- The incidence of chemosis and subconjunctival hemorrhage were significantly lower in GroupLD $(n=15)$ in comparison to GroupWS $(n=26)$ $(p=0.001$ and $p<0.001$, respectively).
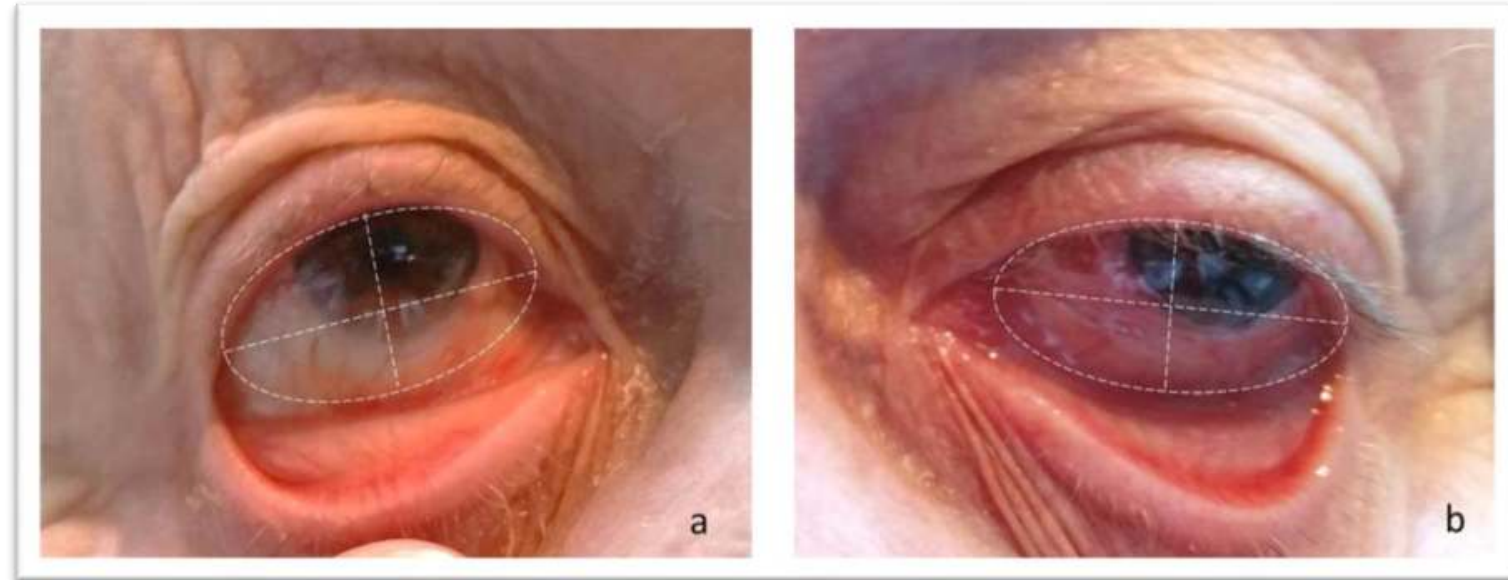

Fig2. Subconjunctival hemorrhage following STB in the same patient; with lacrimal dilator in the right eye (a) and with scissors in the left eye (b)

\section{Conclusions}

Incisionless sub-Tenon's block with lacrimal dilator is a practical and effective alternative to standard technique with reduced chemosis and subconjunctival hemorrhage 